\title{
Ion controlled passive nanoparticle uptake in lipid vesicles in theory and experiment
}

\author{
F. G. Strobl ${ }^{1}$, D. M. Czubak ${ }^{1}$, A. Wixforth ${ }^{1,2,3}$ and C. Westerhausen ${ }^{1,2,3,4}$ \\ ${ }^{1}$ Chair for Experimental Physics I, University of Augsburg, Germany \\ ${ }^{2}$ Nanosystems Initiative Munich, Schellingstraße 4, 80799 Munich, Germany \\ ${ }^{3}$ Center for NanoScience (CeNS), Ludwig-Maximilians-Universität, Munich 80799, \\ Germany
}

${ }^{4}$ Zentrum für Interdisziplinäre Gesundheitsforschung (ZIG), 86135 Augsburg, University of Augsburg, Germany

${ }^{*}$ E-Mail: christoph.westerhausen@gmail.com

\section{Abstract}

Despite the increasing number of applications of nano-sized particles (NP), there is a lack of systematic basic experimental studies on the physical basics of the interactions between NP and cell membranes. Here, we follow a bottom-up approach and investigate the intake of silica NP by Giant Unilamellar Vesicles (GUVs). We observe a massive nanoparticle uptake by fluid phase vesicles, but only above a specific ionic strength of the surrounding buffer solution. The uptake rates increase for decreasing $\mathrm{NP}$ size and increasing $\mathrm{NaCl}$ concentration. A correlation of ionic strength and adhesion force between the lipid membrane and the NP can explain this dependency. We discuss these effects employing a model which considers NP diffusion and an effective membrane permeability due to uptake-induced pores. Our findings contribute to a deeper understanding of the physics behind NP-membrane interactions as well as endocytotic particle uptake in living cells. 


\section{Introduction}

Nowadays, nanoparticles (NP) are not only ubiquitous in the food industry, cosmetics and biomedicine but have also become a promising tool for medical purposes. Due to their size, NP show distinct interaction mechanisms with biological membranes. Similar to biomolecules or viruses, NP can influence and penetrate cell membranes through several uptake pathways ${ }^{1-5}$. Here, we focus on endocytosis-like uptake processes. In this context, the term "endocytosis-like" denotes uptake processes exhibiting two main steps: (I) an envelopment of the particle by the plasma membrane and (II) a subsequent fission process, in which enveloped particles are fully internalized ${ }^{6}$.

Besides the therapeutic use of NP, there are, however, also hazards associated with nanomaterials. NP-induced complications and inflammations during pregnancy have been observed in mice ${ }^{7,8}$. These studies also indicate a significant size dependence of the observed effects. A systematic physical understanding of these processes is not only necessary for a better insight into the uptake machinery of living cells but also an important approach to the optimization of drug delivery systems ${ }^{9,10}$. Gao et al. studied drug transport to mice brains by differently sized, drug-loaded NP. They found significantly higher drug delivery rates for NP with a diameter of $70 \mathrm{~nm}$ compared to larger NP ${ }^{11}$. Using a cell-based in vitro blood-brain barrier (BBB) model, Hanada et al. report a maximum of the BBB permeability for silica nanoparticles of around $30 \mathrm{~nm}$ in diameter ${ }^{12}$.

It is crucial to understand the driving forces of NP uptake into cells through the plasma membrane. As we will show, the observed size effects in cellular particle uptake are not (or at least not solely) a result of active biochemical processes but can be explained by basic physical surface interactions. To elucidate the physical basics of the abovementioned biological mechanisms, we investigate similar - - though entirely passive - interaction processes between NP and lipid vesicles which can serve as universal model systems for biological membranes.

As pointed out in several theoretical publications, for instance Deserno et al. ${ }^{13}$, an antagonism between adhesion forces on the one side and membrane tension and bending forces on the other can control the envelopment of single particles by biological membranes. However, there is still a lack of comparable experimental data on size-dependent uptake and especially on the dynamics of many-particle uptake events. As described elsewhere, in the uptake of a large number of particles by one 
cell or vesicle, the role of membrane tension becomes more important due to a competitive interaction between single particles ${ }^{6}$.

For a deeper understanding of these effects, we here present additional data on uptake dynamics and dependencies on particle concentration and adhesion strength. In particular, we varied the adhesion strength and thus the driving force for NP uptake by adjusting the salt concentration for different particle sizes and concentrations ${ }^{14}$. Indepth experiments on these aspects will be followed by a discussion of a plausible theoretical picture of adhesion-driven particle uptake.

\section{Materials and Methods}

\section{Sample Preparation}

1,2-dioleoyl-sn-glycero-3-phosphocholine (DOPC) dissolved in chloroform was purchased from Avanti Polar Lipids (Alabaster, Alabama, USA), 3,3'ditetradecyloxacarbocyanine (DiOC14) from Biotium Inc. (Hayward, CA, USA), $\mathrm{Na}_{2} \mathrm{HPO}_{4}, \quad \mathrm{NaH}_{2} \mathrm{PO}_{4}$, sucrose and D-(+)-glucose monohydrate from Merck (Darmstadt, Germany). For aqueous solutions, ultrapure water (pure Aqua, Germany) with a specific resistance $\geq 18 \mathrm{M} \Omega$ was used.

GUVs were prepared by electroformation, as first described by Angelova et al. ${ }^{15}$. In short, lipids in the desired ratio and $0.05 \mathrm{~mol} \%$ of the fluorescent marker DiOC14 were mixed in chloroform and spread onto fluorine tin oxide (FTO)-coated glass slides. The solvent was thoroughly removed through vacuum evaporation. For the swelling procedure, a chamber was assembled from two of the slides and a spacer filled with $150 \mathrm{mM}$ sucrose solution. An AC voltage was applied for a minimum of 4 hours $(f=$ $10 \mathrm{~Hz}, E_{\text {eff }}=0.6 \mathrm{~V} / \mathrm{mm}$ ) at room temperature. The osmolarity of all solutions was measured with an Osmomat 030 (Gonotec, Germany) and adjusted to $150 \mathrm{mM}$.

Monodisperse, non-porous silica nanoparticles with silanol groups on the NP surface were purchased from nanoComposix (Prague, Czech Republic). Particle size distributions, surface area as well as the $\zeta$-potentials were given by the manufacturer. These data can be found in Table 1. 
Table 1: Physical parameters of the silica nanoparticles

\begin{tabular}{llll}
\hline Name & Diameter $(\mathrm{nm})$ & ל-potential $(\mathrm{mV})$ & surface area $\left(\mathrm{m}^{2} / \mathrm{g}\right)$ \\
\hline $20 \mathrm{~nm}$ particles & $21.9 \pm 2.8$ & -16.1 & 120.6 \\
$50 \mathrm{~nm}$ particles & $48.1 \pm 5.3$ & -53.2 & 55.4 \\
$60 \mathrm{~nm}$ particles & $57.8 \pm 3.5$ & -44.7 & 46.8 \\
$80 \mathrm{~nm}$ particles & $82.6 \pm 4.7$ & -32.7 & 32.8 \\
$140 \mathrm{~nm}$ particles & $142.4 \pm 8.1$ & -42.6 & 18.5 \\
\hline
\end{tabular}

The particles were centrifuged and re-dispersed in ultrapure water twice to ensure high purity. The initial particle surface area concentration $C^{A}$ (sum of the surface area of all particles in solution, $\left.C^{\mathrm{A}}=C\left(4 \pi r^{2}\right)\right)$ was taken from the datasheets of the manufacturer and was adjusted to the values given in the results section. The medium used for the experiments was a HEPES buffered solution of glucose and $\mathrm{NaCl}$. The $\mathrm{pH}$ was adjusted to a value of $\mathrm{pH}=7$. The osmolarities of medium and GUV-containing sucrose solutions were matched by the addition of glucose to prevent osmotic tension in the vesicle membrane.

Cover glasses, which were used as observation plane, were coated with a film of agarose to prevent unspecific adhesion of the GUVs to the glass surface, which could, of course, influence the membrane tension. For this purpose, agarose was first dissolved in boiling water at a concentration of $25 \mathrm{mg} / \mathrm{ml}$ and then spin-coated onto $\mathrm{O}_{2}$-plasma cleaned microscope cover slides. The film was then dried on a hotplate at $60^{\circ} \mathrm{C}$.

\section{Experimental procedure}

A coated cover glass was fixed onto a microscope slide using double-sided adhesive tape with a thickness of about $200 \mu \mathrm{m}$ to form a capillary chamber. GUVs and NP suspensions were gently mixed in the ratio of $195 / 5$ and used to fill the observation chamber. Due to the density difference between glucose and sucrose, the GUVs sank to the bottom of the observation chamber. The colloidal stability of the particles was confirmed by dynamic light scattering (DLS) for each experimental condition using a 90Plus Particle Size Analyzer (Brookhaven Instruments Corporation, New York, NY, USA, temperature $20^{\circ} \mathrm{C}$, viscosity $1.002 \mathrm{cP}$, ref. index fluid 1.331 , angle $90^{\circ}$, wavelength $666 \mathrm{~nm}, 5$ Runs á $2 \mathrm{~min}$, ref. index real 1.550). Unless otherwise specified, the particle concentration was adjusted to a surface area concentration of $C^{\mathrm{A}}=$ 
$0.5 \mathrm{~m}^{2} / \mathrm{I}$. The temperature in the observation chamber was stabilized to $T=20^{\circ} \mathrm{C}$ by a PID-controlled Peltier element embedded in an aluminum holder containing the observation chamber.

As discussed in previous work ${ }^{6}$, single particle uptake can be ingested by GUVs through an endocytosis-like mechanism as described below. During this process, membrane area is "consumed" and, as a consequence, the vesicle shrinks. The decrease in vesicle cross-sectional area was observed by fluorescence microscopy (Zeiss Axiovert 200M, Hamamatsu Orca G) and analyzed by a custom Image-J script. Since the vesicles maintain a spherical shape during the entire uptake process, the cross-sectional area can be converted directly into the vesicle surface area $A(t)$.

\section{Model development}

\section{General description of the "endocytosis-like" uptake mechanism}

In our previous work ${ }^{6}$, we briefly discussed a model describing the simultaneous uptake of many single particles by a vesicle. As shown in Figure 1, we expect this process to be initiated by unspecific surface adhesion, represented by a contribution to the specific free energy per membrane area $g_{a d h}<0$ and moderated mainly by vesicle tension and bending stiffness, i.e. the specific free energy per membrane area $g_{\text {ten }}>0, g_{\text {ben }}>0$. 

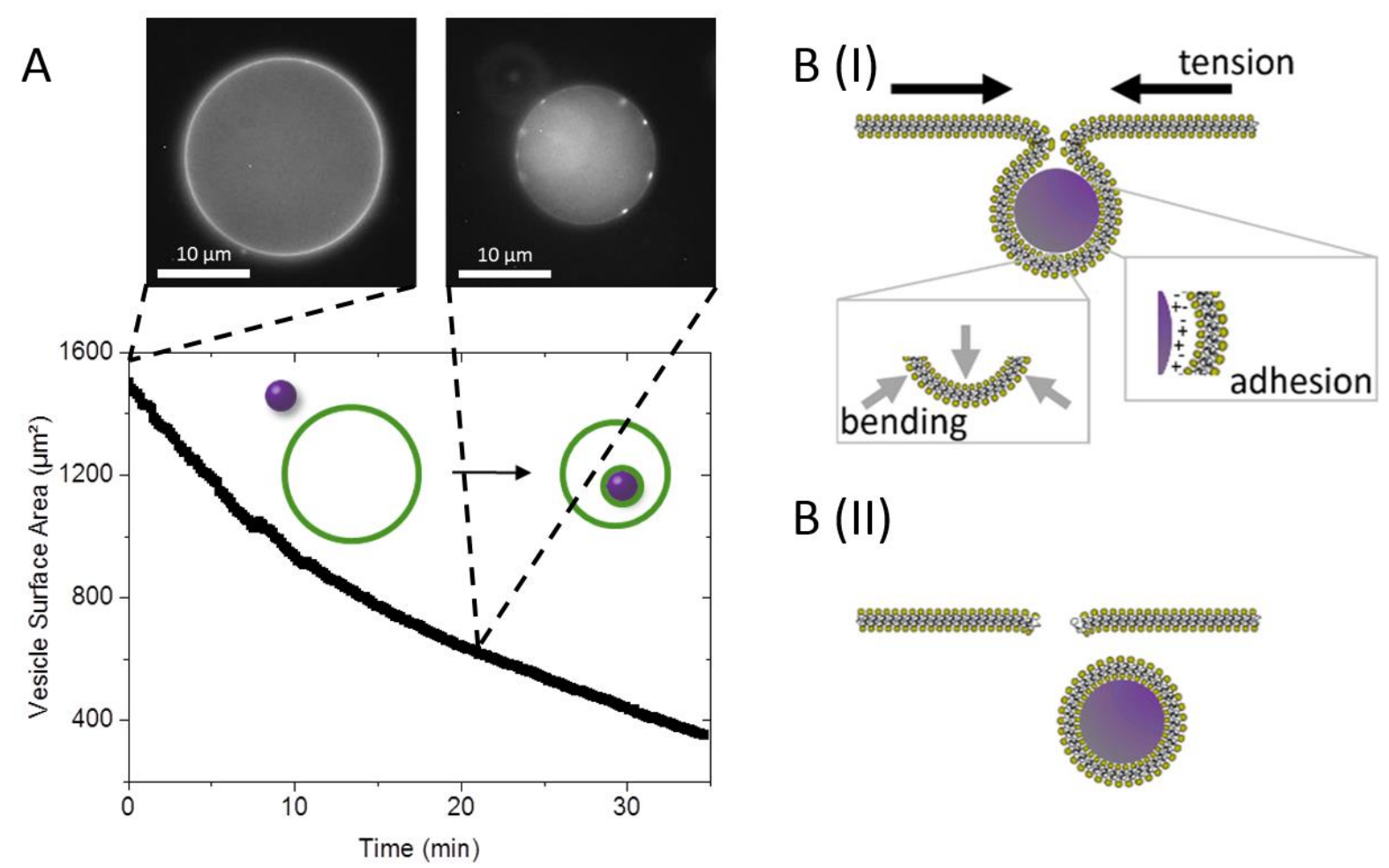

B (II)

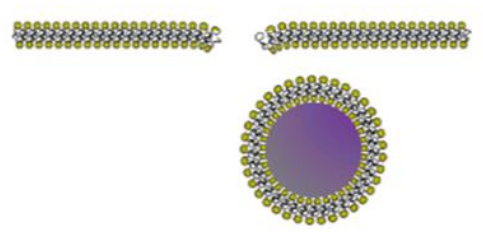

FIGURE $1 \mathrm{~A}$. Typical graph $\boldsymbol{A}(\mathrm{t})$ for the shrinking of a GUV during particle uptake and two micrographs at $t=0 \mathrm{~min}$ and $\mathrm{t}=\mathbf{2 0} \mathrm{min}$. $\mathrm{B}(\mathrm{I})$. Illustration of NP envelopment by a lipid bilayer during NP uptake. The three energetic contributions for NP uptake are indicated, namely tension (caused by membrane imprinting), bending (due to the envelopment of the NP) and adhesion induced by a diluted ionic environment. B(II). NP uptake is completed after full envelopment of the NP. The resulting pore heals within a typical lifetime ${ }^{6}$.

Figure $1 \mathrm{~B}(\mathrm{I})$ and $1 \mathrm{~B}(\mathrm{II})$ illustrate the uptake process. An NP adheres to the membrane and becomes enveloped by the membrane until the neck of the membrane breaks and releases the NP into the interior of the vesicle ${ }^{16-22}$. Such a nanoparticle uptake will leave a transient membrane defect. Current models for membrane pore dynamics predict the existence of a metastable state for membrane pores with a typical diameter $R_{p}$ of few $\mathrm{nm}$ and a typical lifetime $\tau$ of a few milliseconds, once a critical defect size is reached ${ }^{23-25}$. The metastable value for $R_{p}$ is found to depend only weakly on the membrane tension. The lifetime, however, can be significantly prolonged by a membrane tension $\sigma>0$.

It should be mentioned that once a certain critical membrane tension is exceeded, such transient pores can become instable and lead to transient or destructive membrane break down ${ }^{26,27}$. Since we have never observed any particle expulsions in our experiments, we expect all pores to be in a metastable state. 


\section{Modelling uptake kinetics}

DOPC GUVs were chosen as a representative system for fluid membranes. We investigated particle uptake under varying $\mathrm{NaCl}$ concentrations from $0 \mathrm{mM}$ to $90 \mathrm{mM}$. NP of different diameters (see Table 1 for further information) and concentrations $\left(0.5-4.0 \mathrm{~m}^{2} / \mathrm{I}\right)$ were used.

We have shown previously that for ion concentrations above a critical value, vesicles shrink in diameter due to NP uptake ${ }^{6}$. The change of vesicle surface area $A(t)$ is proportional to the number of internalized particles. A measurement of the vesicle surface area during particle uptake yields typical shrinkage curves as depicted in Figure $1 \mathrm{~A}$. Typically, the function $A(t)$ is rather complex and depends on various parameters which influence each other during NP uptake, as described in the following section.

For the analysis of $A(t)$ we follow a simple empirical model of the uptake dynamics. We assume that two main mechanisms can determine the uptake rates and in consequence vesicle shrinking. Firstly, there will be a typical timescale for the permeation of a particle through the membrane. Secondly, the diffusion of NP from the surrounding NP reservoir to the vesicle will lead to the development of a depletion zone, i.e. a reduced relevant particle concentration near the vesicle surface. This situation is shown in Figure 2. For a given NP concentration $C_{\mathrm{s}}$ in the vicinity of the vesicle, the rate of NP uptake depends on the membrane's ability to deliver surface area. In a simplifying approximation, we describe the penetration of the membrane as a diffusive process. The uptake is thus limited by an effective permeability $P$ of the membrane and by the NP concentration at the vesicle surface $C_{\mathrm{s}}$. 


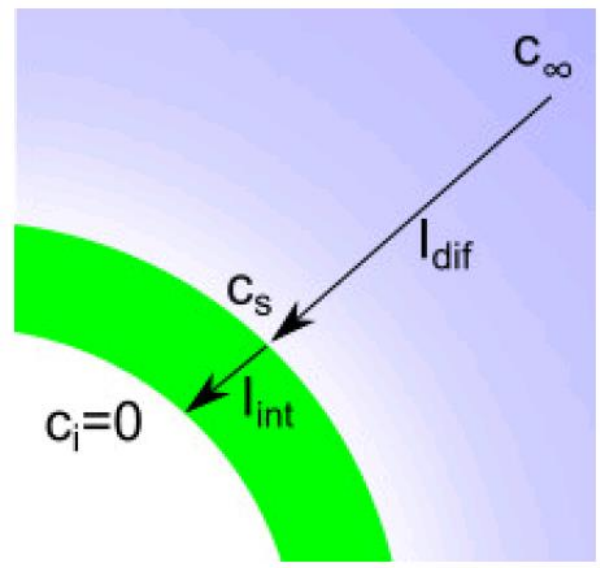

FIGURE 2 Due to NP uptake, a depletion zone develops in the vicinity of the vesicle's surface, leading to a concentration gradient. This gradient results in a diffusion current ldif towards the vesicle. The NP uptake itself is described empirically by an internalization current $l_{\text {int. }}$.

As shown in the supporting information, the following expression for the time dependent area of the vesicle $A(\mathrm{t})$ as function of membrane permeability, diffusion constant and nanoparticle concentration can be derived.

$$
A(t)=\frac{4 \pi D^{2} \mathfrak{B}\left(\frac{\sqrt{\frac{e^{\frac{K}{D \sqrt{\pi}}-C_{\infty}^{A} P t} P^{2}}{D^{2}}}}{2 \sqrt{\pi}}\right)^{2}}{P^{2}}
$$

with $K$ as an integration constant and the Lambert-W-function $\mathfrak{M}$.

Equation (1) can now be used to fit the experimental data. If either particle diffusion or membrane permeability dominate the process, we can approximate Equation (1) by much simpler expressions.

Therefore, we introduce a dimensionless parameter $X$ to characterize the influence of the two limiting mechanisms:

$$
X:=P \sqrt{A} / D
$$

For $X \gg 1$, NP uptake behavior depends primarily on the diffusion of NP towards the vesicle, whereas for $X \ll 1$ membrane permeability plays the major role. Both contributions have to be taken into account for $X \approx 1$.

For $X \ll 1$, Equation (1) simplifies to:

$$
\frac{\mathrm{d} A}{\mathrm{~d} t}=-C_{\infty}^{\mathrm{A}} P A(t)
$$


and therefore

$$
A(t)=A_{0} \mathrm{e}^{-C_{\infty}^{\mathrm{A}} P t} \stackrel{t \rightarrow 0}{\longrightarrow} A_{0}-C_{\infty}^{A} A_{0} P t
$$

with the initial surface area $A_{0}=A(t=0)$.

In contrast, for $X \gg 1$ it follows that:

$$
\frac{\mathrm{d} A}{\mathrm{~d} t}=-2 \sqrt{\pi} C_{\infty}^{\mathrm{A}} D \sqrt{A(t)}
$$

leading to the solution:

$$
A(t)=A_{0}-2 \sqrt{\pi A_{0}} C_{\infty}^{\mathrm{A}} D t+\pi C_{\infty}^{\mathrm{A}^{2}} D^{2} t^{2} \stackrel{t \rightarrow 0}{\longrightarrow} A_{0}-2 \sqrt{\left(\pi A_{0}\right)} C_{\infty}^{A} D t
$$

This solution corresponds to a convex parabola with its apex on the time-axis. Obviously, only the left side $(\mathrm{t} \leq 0)$ of the parabola is physically relevant. After reaching $A=A_{0}$, the vesicle surface area remains constant. For short observation times $t$, it is in both cases sufficient to employ a linear fitting function.

Thus, the experiments shown in Figure 1 allow the determination of uptake rates which can be interpreted as permeability $P$, as long as only a small fraction of impacting particles is internalized. As soon as the uptake becomes very efficient, the uptake rate should be a measure of the particle diffusion constant.

\section{Energy considerations regarding NP size \& ionic environment}

The membrane permeability $P$ is a consequence of the antagonism between different energy contributions, i.e. the thermodynamic forces controlling the single particle uptake. The basic free energy contributions in our model are the adhesion energy per unit area $g_{\text {adh }}$ on the one hand and its competitors on the other, namely bending energy

$g_{\text {ben }}$ and $g_{\text {ten }}$, determining the free energy necessary for the expansion of the membrane against its surface tension ${ }^{28}$. For NP envelopment, these contributions have to fulfill:

$$
g_{\text {adh }} \geq g_{\text {ben }}+g_{\text {ten }}
$$


For spherical NP and a given bending modulus of about $\kappa=10^{-19} \mathrm{~J}$ for fluid membranes with negligible spontaneous curvature ${ }^{29}$, Helfrich's expression for the bending energy per unit area $g_{\text {ben }}$ can be simplified to ${ }^{30}$ :

$$
g_{\mathrm{ben}}=2 \kappa / r_{\mathrm{NP}}^{2}
$$

Our particle radii imply bending energy densities in the range from $0.04 \mathrm{~mJ} / \mathrm{m}^{2}$ to $2.00 \mathrm{~mJ} / \mathrm{m}^{2}$ for radii between $70 \mathrm{~nm}$ and $10 \mathrm{~nm}$.

At a first glance, the bending energy would thus favor the uptake of larger NP compared to smaller ones. However, the uptake of larger NP leads to higher membrane tension during the uptake of a single NP. The question arises of how bending and tension account for the energy contribution from Equation 7. In our experiments, we find the uptake to be more efficient the smaller the particle size is. Therefore, we expect $g_{\text {ten }}$ to be the dominant energy contribution controlling the uptake process.

As described in our previous work ${ }^{6}$, and as also found in our recent experiments, membrane tension can indeed stop further uptake after the intake of a certain number of particles. However, above the adhesion energy density $g_{a d h}^{*}(R)$, we observe unlimited particle uptake and vesicle shrinkage until a vesicle size below the resolution limit of our system. Since we never observe escaping particles, we hypothesize that the membrane tension (i.e. vesicle pressure) is released via particle-induced pores no larger than $15 \mathrm{~nm}$ in diameter.

Dietrich et al. ${ }^{21}$ describe how each NP adhering to the vesicle creates membrane tension depending on the state of penetration, quantified by a penetration parameter $z$ ( $z=0$ indicating no penetration, and $z=2$ indicating full envelopment). The more NP are internalized, the more vesicle surface area $A$ is consumed, where $A_{\text {eq }}$ is the surface area at zero tension. These considerations imply conservation of volume as follows: $A-A_{\text {eq }}=\pi r^{2} z^{2}-A_{\text {eq }} \varepsilon$, where $\varepsilon \geq 0$ is the relative area excess. Deserno et al. extended Dietrich's model of membrane tension by including membrane bending induced by a single particle and set the theoretical background to our model ${ }^{13}$. For our analysis, we simplify this model as follows:

- We neglect the "neck" energy contribution during partial particle envelopment.

- We neglect the bending energy contribution from the initial membrane curvature of the vesicle, since $R>>r$ for all our experiments. 
For this case Deserno et al. give an expansion for the phase boundary between partial and full envelopment of one particle:

$$
\frac{r}{R_{0}}=x^{1 / 2}-\frac{1}{3} x+\frac{5}{18} x^{\frac{3}{2}} \mp \cdots \text { with } x=\frac{\zeta / 2+\varepsilon}{1-\varepsilon}(9)
$$

In our previous work ${ }^{6}$, we gave an estimation for the maximum number $\mathrm{N}$ of internalized particles without any pore formation, i.e. without any pressure release mechanism:

$$
\varepsilon(N)=\frac{A_{e q}-A}{A_{e q}}=\frac{\left(1^{2}-N r^{2}\right)-\left(R^{3}+N r^{3}\right)^{\frac{2}{3}}}{\left(R^{3}+N r^{3}\right)^{2 / 3}}(10)
$$

where $R$ describes the initial vesical radius.

Instead of a deeper analysis of single particle uptake, we now look into the manyparticle uptake scenario.

If we consider a fission process and subsequent pore formation, more particles can be internalized until the threshold number of internalized particles is reached. As soon as the pressure release per pore is "faster" than the pressure-rise due to particle uptake, the threshold number will approach infinity, resulting in a new phase of particle uptake. Thus, we envision a phase diagram of particle uptake with four phenomenological phases (see Figure 3):

1) Particle uptake is impossible, if the condition in Equation 7 is not fulfilled

2) Only partial uptake of particles is possible within the boundary described by Equation 9

3) Above this boundary, a limited number of particles can be completely enveloped

4) Additionally to these phases already covered ${ }^{13}$, we hypothesize an additional phase of "unlimited" particle uptake, where particle uptake and subsequent volume loss through induced pores leads to vesicle shrinking.

The boundaries of these phases can be found by numerical computation, based on the above conditions. Therefore, we need a model for pressure release through induced pores.

Our first assumption is that each internalized particle induces a pore. The size of this pore will be determined by the interplay between surface and line tension. At a given pore radius $R_{\mathrm{p}}$ smaller than the membrane thickness of typically $d=3 \mathrm{~nm}$, we can roughly describe the volume flow $\dot{V}$ through the pore by the Hagen-Poisseuille law ${ }^{31}$ : 


$$
\dot{V}=\frac{\pi R_{p}^{4} \quad p}{8 \eta d}=\frac{\pi R_{p}^{4} \quad \sigma}{4 \eta R d}
$$

where $\eta$ denotes the viscosity of the fluid inside the vesicle and $p$ the overpressure generated by particle uptake. The second identity thereby follows from the YoungLaplace equation for a vesicle with radius $R$ and surface tension $\sigma$. If we now assume an effective pore life time $\tau$, it follows that for the mean volume loss through each pore:

$$
\Delta V=\frac{\pi R_{p}^{4} \quad \sigma \tau}{4 \eta R d}(12)
$$

We perform a time-discrete simulation, assuming particles arriving at a fixed rate per surface area. For each particle, the uptake possibility is evaluated according to the above criterion (Equation 9). Each uptake event induces a pore and the volume loss through the pores is calculated. Finally, the number of closing pores is calculated assuming a Poisson type probability distribution for pore closing given by the ratio of time step size and pore lifetime. Based on this scheme a full phase diagram of particle uptake can be derived.

If we look at two phase diagrams of constant pore parameters $\tau$ and $R_{p}$, we end up with 2-dimensional phase diagrams corresponding to a simplified version of the phase diagram in Desermo et al. ${ }^{13}$ (see Figure 3). However, the dark area represents the new phase of unlimited particle uptake.

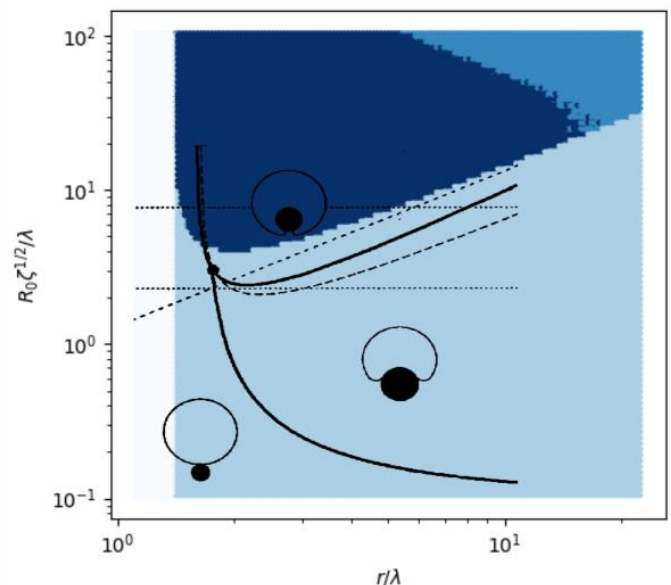

(a)

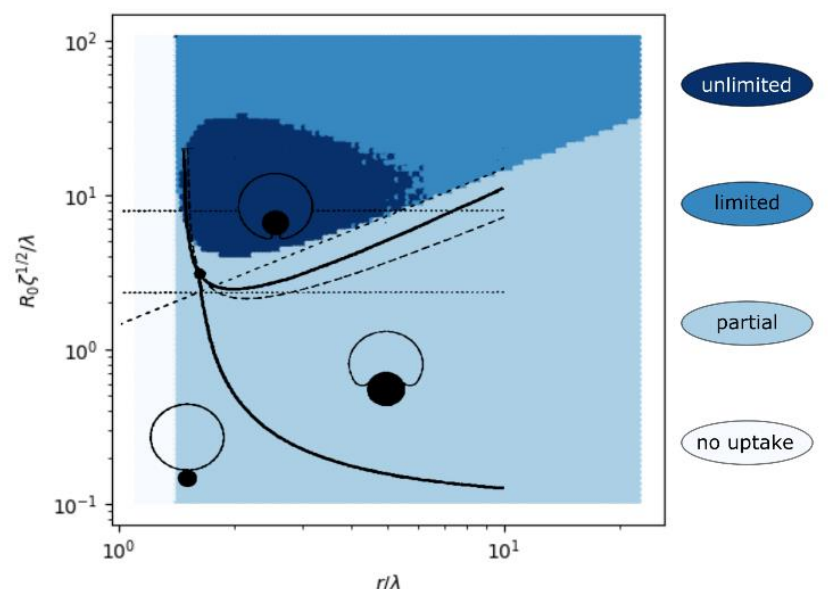

(b)

FIGURE 3 Plane phase diagrams for $\tau=500 \mathrm{~ms}$ and pore radii $R_{p}=5 \mathrm{~nm}$ (a) and $R_{p}=2 \mathrm{~nm}$ (b). The black overlay is adapted from Desermo et al. ${ }^{13}$. Obviously, phases 1, 2 and 3 correspond in both models except for very high tensions or very small vesicle radii. The differences can be explained by the simplifications 
explained in the text. We also see a new phase of unlimited particle uptake developing with longer pore lifetime.

This allows for the examination of the minimal adhesion strength $g_{a d h}^{*}(r)$ for unlimited particle uptake, holding all other parameters constant at typical values for DOPC giant vesicles (see Table 2). 
Table 2: Simulation parameters

\begin{tabular}{ll}
\hline Parameter & Value \\
\hline Bending rigidity & $4 \times 10^{-20} \mathrm{~J}^{32}$ \\
Area compression modulus & $0.23 \mathrm{~N} / \mathrm{m}^{33}$ \\
Membrane thickness (hydrophobic core) $d$ & $2.8 \mathrm{~nm}^{34}$ \\
Pore radius $R_{\mathrm{p}}$ & $9 \mathrm{~nm}$ \\
Pore life time $\tau$ & $500 \mathrm{~ms}$ \\
\hline
\end{tabular}

Figure 4 shows the results, where the regime of unlimited particle uptake corresponds to the dark blue area. For a given adhesion strength $g_{\text {adh }}$, we expect a lower threshold to the particle size due to bending limitation, as well as an upper threshold due to tension limitation. "Real" unlimited uptake i.e. uptake until complete vesicle collapse, is extremely energy-consuming for large particles, as shown in Figure 4a. In our experiments, however, we observe vesicles over a limited time, typically $20 \mathrm{~min}$. A limited uptake of a very large number of particles cannot be discriminated from an unlimited uptake. To account for this, Figure $4 \mathrm{~b}$ shows the simulation result for an alternative "apparently unlimited" uptake, when the uptake is strong enough to induce a shrinkage of the vesicle surface area of $>1 \%$.

In the experiments described further below, we investigate a range of particle sizes between $r=10 \mathrm{~nm}$ and $r=70 \mathrm{~nm}$, i.e. we would expect to mainly observe the upper threshold in our experiments. The lower threshold was unfortunately not accessible in our experiments due to the size limits of the round silica particles available. To test the existence of a lower threshold, other model systems would have to be used. Moreover, other studies have shown that very small particles can directly penetrate the lipid membranes. In this case, our model would not be relevant. 


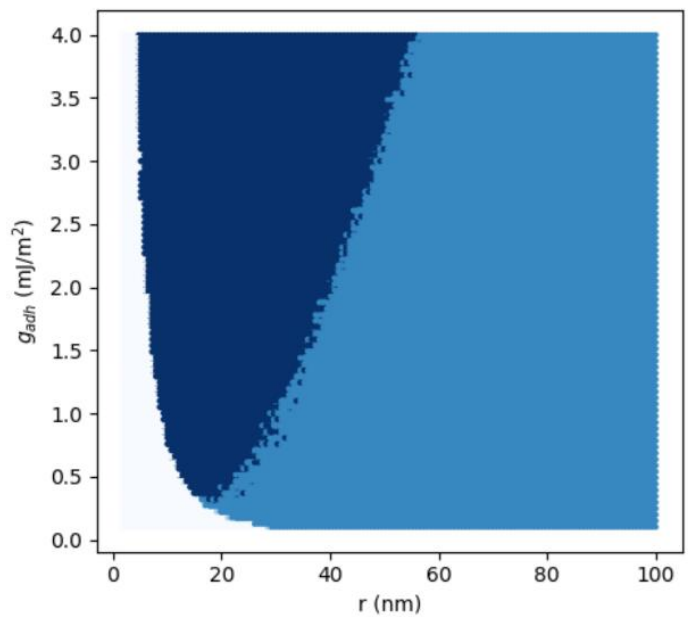

(a)

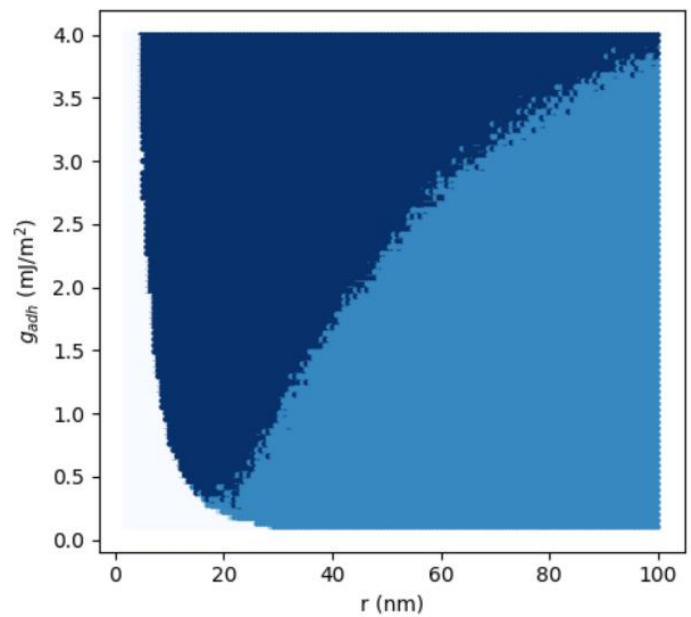

(b)

FIGURE 4 Phase boundaries for unlimited uptake for varying particle size and adhesion strength $g_{\text {adh }}$. (a) Real phase boundary for unlimited uptake (b) apparent phase boundary if a shrinkage of $>1 \%$ of the initial vesicle surface area is already considered as unlimited case (see table 2 for simulation parameters). 


\section{Experimental Results \& Discussion}

Particle-size-dependent correlation between uptake rate and ionic concentration

For the system studied in this work, a specific ionic environment is prerequisite in order to observe passive NP uptake. It has been shown that adhesion strength between silica particles and lipid membranes is strongly correlated to the ion concentration of the surrounding medium ${ }^{35,36}$. In the following section, we quantitatively determine the influence of salt concentration on the NP uptake. Fitting the experimentally determined values $A(\mathrm{t})$ using Equation (4), we obtain a value for the membrane permeability $P$. Figure 5 shows the shrinking rate $\frac{1}{A_{0}} \frac{d A}{d t}$ as function of $\mathrm{NaCl}$ concentration for different NP diameters.
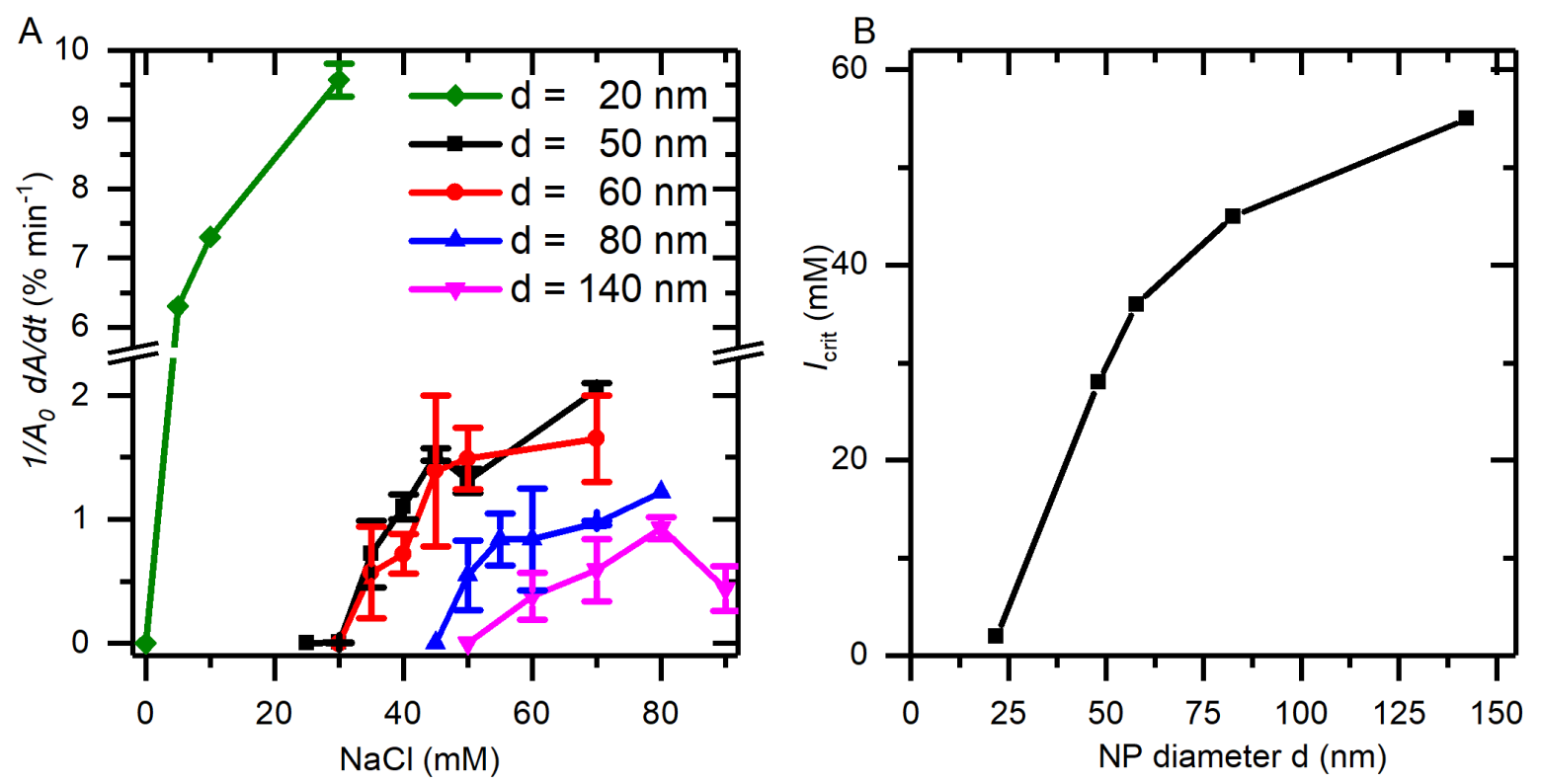

FIGURE 5 A. NP uptake rates into DOPC vesicles as a function of $\mathrm{NaCl}$ concentration. The larger the NP, the lower the uptake rate as expected from the model discussed in the text. For one particle size, the uptake rises monotonically with ion concentration. The exception for $140 \mathrm{~nm}$-particles can be explained by colloidal instability of the particle suspension. For slightly higher ionic concentration, strong particle flocculation was observed. B. The minimal salt concentration $I_{\text {crit }}$ for observing NP uptake as function of NP diameter increases with increasing size. There is a good qualitative agreement with apparent phase boundary predicted from the model (see Figure $4 \mathrm{~b}$ ).

For all NP sizes, we find a systematic correlation between uptake rate and salt concentration. Below a threshold $I_{\text {crit }}$, no NP uptake takes place. Above $I_{\text {crit }}$, the observed uptake rates increase and approach a distinct saturation value $P_{\max }$. This transition is much sharper for small particles than for large particles. The DOPC vesicles studied here show the highest permeability for the smallest NP used $\left(r_{N P}=\right.$ $10 \mathrm{~nm}$ ). Even for very low salt concentrations, significant particle uptake can be 
observed. For larger NP, an increasing minimal salt concentration $I_{\text {crit }}$ up to $60 \mathrm{mM}$ is necessary, as shown in Figure 5B. There is no systematic study on NP size and salt concentration for uptake of silica NP in phospholipid membranes, but our results are consistent with the few data available in the literature: Le Bihan et al. reported size dependent, endocytosis-like uptake of comparable silica NP in DOPC GUVs ${ }^{37}$. While NP with diameters between $d=190 \mathrm{~nm}$ and $d=30 \mathrm{~nm}$ are fully enveloped by the membrane, smaller NP with a diameter $d=15 \mathrm{~nm}$ only adhere to the outer layer of the membrane. These experiments were done in $150 \mathrm{mM} \mathrm{NaCl}$, i.e. the high adhesion energy case. Moreover, Michel et al. report on the uptake of small silica NP ( $d=16$ $\mathrm{nm}$ ) in small unilamellar DOPC vesicles ${ }^{38}$. To the best of our knowledge, in these experiments no $\mathrm{NaCl}$ was added. However, as these particles are even smaller than the smallest particles studied here, even traces of salt could lead to NP uptake as indicated by our experiments for NP with $d=20 \mathrm{~nm}$. Finally, a theoretical study by Smith et al. concludes that when the adhesion strength is increased above a threshold value, the membrane fully envelops the particle, but that the NP remains tethered to the membrane if the membrane is homogeneous. In that study the authors claim that non-adhesive domains, i.e. an inhomogeneous membrane, are necessary for the rupture of the membrane neck. While the first finding is in agreement with our data, the latter contradicts our data and earlier work ${ }^{38,6}$.

For an analysis of the values $I_{\text {crit }}$ and $P_{\max }$ we fit the empirical function

$$
P(I)=P_{\max }\left(1-\exp \left(a\left(I-I_{\text {crit }}\right)\right)\right.
$$

to the curves presented in Figure 5.

According to our hypothesis, the saturation measurement value $P_{\max }$ will not actually be determined by the membrane properties, but by limited particle diffusion. That is, if we fit the experimental data for maximum uptake with Equation (6) with $D$ as single fitting parameter, we would expect $D$ to be near the theoretical value derived from the Stokes-Einstein equation:

$$
D=\frac{k_{B} T}{6 \pi \eta r}
$$

Figure 6 shows a very good correspondence between theoretical and experimentally derived values. We find the strongest deviations for very small particles. One explanation could be a slight overestimation of the hydrodynamic radius due to artifacts 
in the DLS measurements induced by particle agglomerates. Nevertheless, the good agreement suggests that the uptake efficiency of the membrane can be close to infinity for a limited number of impacting particles.

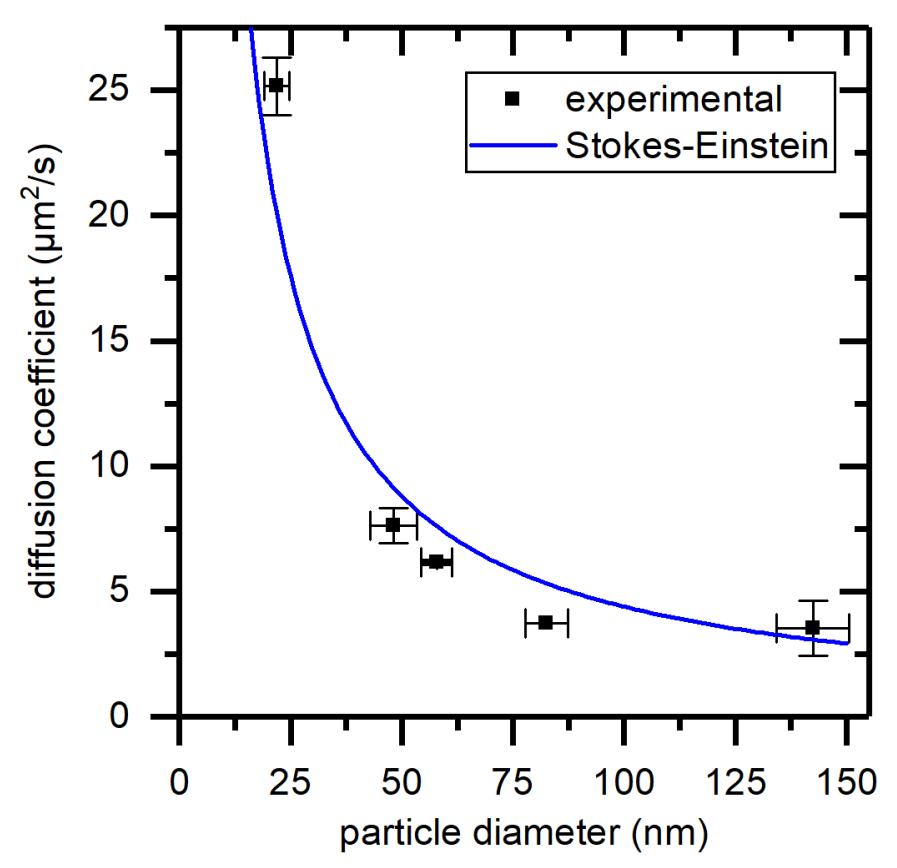

FIGURE 6 Comparison between experimentally derived diffusion coefficient (Equation 8) and the theoretical value predicted from Stokes-Einstein ( $\mathrm{T}=300 \mathrm{~K}, \eta=1 \mathrm{mPas}$ ). The very close correspondence suggests that the observed maximum for the particle uptake rate is dominated by particle diffusion for all particle sizes investigated. 


\section{Suppression of NP uptake at high NP concentration}

So far, we have considered the uptake efficiency to be independent of the particle concentration. However, for very high particle impact rates, we would expect membrane tension to be an obstacle to particle envelopment, especially for large particles. To test this hypothesis, we determined $P(c)$ for $60 \mathrm{~nm} \mathrm{NP}$ at a constant ionic concentration of $50 \mathrm{mM}$ at $\mathrm{T}=20^{\circ} \mathrm{C}$. The resulting vesicle shrinking curves are shown in Figure 7.
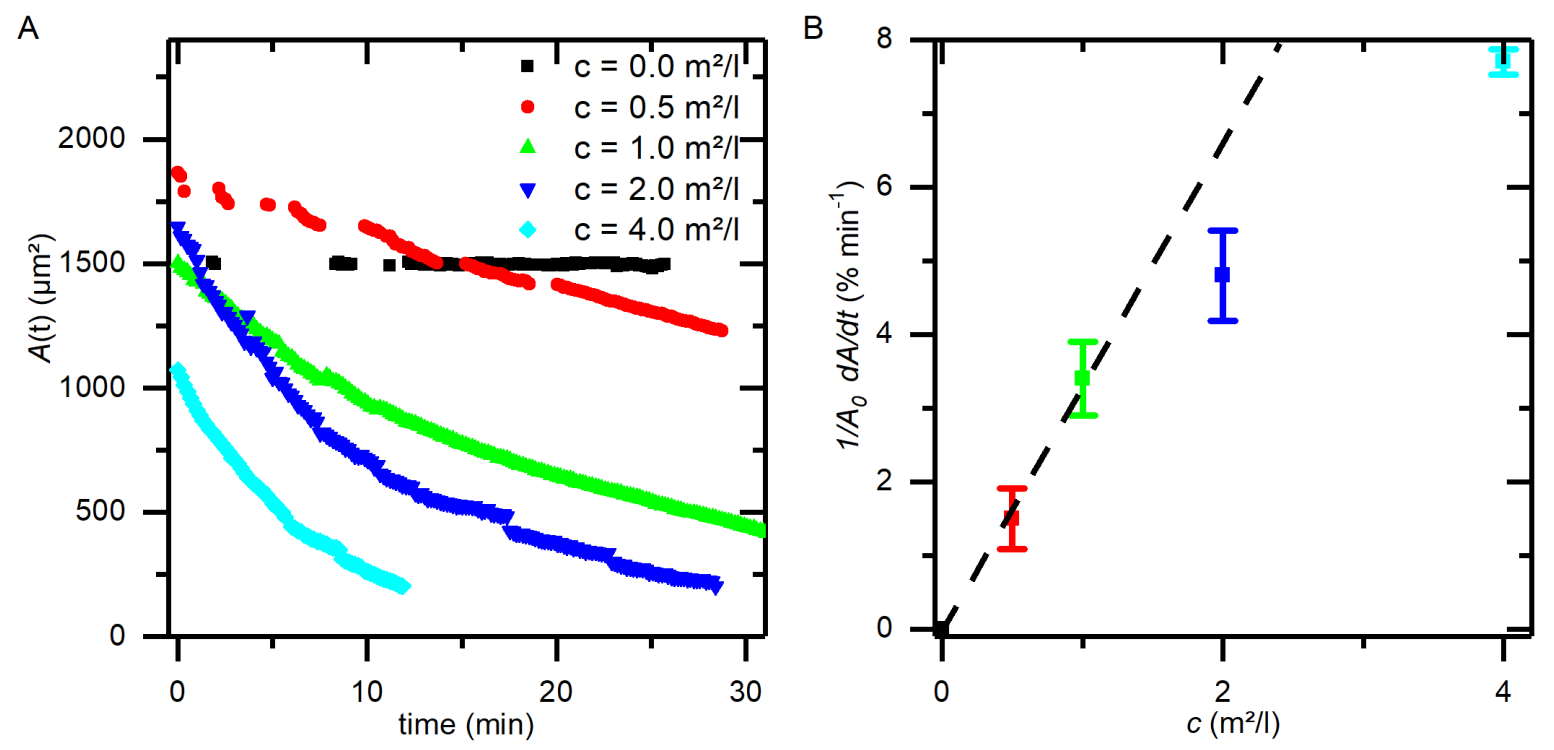

FIGURE 7 (A) Shrinking of DOPC GUVs in the presence of different concentrations of $60 \mathrm{~nm}$ NP at constant salt concentration. (B) The maximal vesicle permeability $P$ determined from $A$ as function of NP concentration. The non-linear relationship can be interpreted as a competitive behavior of NP on the membrane.

For low NP concentrations $c \leq 1 \mathrm{~m}^{2} / \mathrm{I}$, the uptake rate depends linearly on the NP concentration, as expected from Equations (8) and (10). Above $1 \mathrm{~m}^{2} / \mathrm{I}$, the increase in uptake rate becomes non-linear with respect to NP concentration (Figure 7B).

As mentioned above, the most reasonable explanation for the suppressed uptake rates in the high concentration regime (Figure 7) is a tension-mediated competitive behavior. In our explanation, at $I_{\text {crit }}$ the adhesion force reaches a threshold exceeding the sum of the membrane tension and the membrane bending. Our experiment reveals a nonlinear increase of $I_{\text {crit }}$ for larger NP (Figure 6B). We assume that this increase of $I_{\text {crit }}$ corresponds to the increased adhesion forces necessary to overcome the contrary bending and tension forces for larger particles. This cannot be explained by bending forces, as bending energy density decreases for larger particles. Therefore, membrane 
tension must dominate the uptake process and is the major force that adhesion has to overcome in order to result in NP uptake. This has to be rather a membrane mediated particle-particle interaction effect ${ }^{28,40,41}$. However, Michel et al. attribute similar effects observed in small unilamellar vesicles to electrostatic interactions ${ }^{38}$.

More adhered and/or enveloped NP result in an even higher membrane tension. This is caused by a disturbance of the equilibrium state between tension-releasing pore formation and tension-inducing NP adherence. The low number of pores compared to adhered NP is not able to compensate for the high tension, as stated in the theory section. Ergo, membrane tension rises resulting in a decreasing membrane permeability. Overall, taking these considerations into account, membrane tension is very likely to dominate the uptake behavior in the entire particle size regime as long as the system consists of many NP interacting with a vesicle.

\section{Conclusion}

This study describes an endocytosis-like internalization of nanoparticles into liquid phase Giant Unilamellar Vesicles. Uptake rates depend on nanoparticle adhesion and size as well as the membrane's resistance to pore formation, which results in a finite susceptibility to particle uptake. We proposed a simple model, taking into account the formation of pores by membrane fission during a many-particle uptake. This model predicts a system behavior that features many qualitative aspects of the experimental results.

We show the existence of two distinct phases of particle uptake, which we call "limited" and "unlimited" uptake. Experimentally, we focused on the unlimited case and observed an upper threshold for the particle size for given system parameters and find smaller NP to be internalized more efficiently than larger NP.

The size threshold for unlimited uptake is a function of the adhesion force between particle and vesicle surface and can be described qualitatively by the limited volume loss through induced membrane pores. For a conclusive quantitative description, however, there is still too little information on the relation between ion environment and particle-membrane adhesion, as well as on the effect of ions on the membrane permeability. A lower threshold for the particle size could not be observed in this work, but would be expected for experiments on particles with smaller size if a cooperative uptake (as observed in other studies) can be avoided. 
As soon as the adhesion strength crosses said threshold, a relatively sharp transition to unlimited uptake is observed. In this case, the uptake rate is only limited by the diffusion of particles to the vesicle.

We emphasize here that the observed behavior is not only interesting for the development of lipid-particle hybrid systems ${ }^{42}$, but will also to some extent influence cellular uptake mechanisms. The observed and predicted size effects coincide qualitatively and quantitatively with numerous observations in biological systems ${ }^{43-45}$ where a size optimum of typically $10 \mathrm{~nm}<r<40 \mathrm{~nm}$ has been found for particle uptake.

Of course, particle uptake in cells is usually regulated by active processes and influenced by many specific aspects. For example, the cytoskeleton will introduce a shear resistance to the membrane and particles will usually be covered by a protein corona which modifies the interaction forces between particle and cell surface ${ }^{46}$.

However, since typical adhesion forces and mechanical membrane properties of cell membranes and lipid vesicles are similar in many respects, the influence of fundamental physical aspects can be more important than is often expected. Especially, they can provide an explanation for phenomena such as the massive uptake of unspecific nanomaterials such as silica and metal particles and the existence of general size optima for such uptake processes. Moreover, it has been shown before that the thermodynamic state of lipid membranes can switch particle uptake states ${ }^{6}$. Taken together, we believe that lipid related effects can play a major role for particle uptake in living systems and even lipid-controlled uptake mechanisms seem feasible. 


\section{Author Contributions}

DMC carried out experiments, analyzed data and wrote the manuscript. FGS designed the study, carried out the experiments, analyzed the data, performed the simulations and wrote the manuscript. AW participated in the design of the study and helped to draft the manuscript. CW designed and coordinated the study, analyzed data and wrote the manuscript. All the authors read and approved the final manuscript.

\section{Acknowledgments}

The authors thank the DFG for supporting this work within SPP1313. Moreover, the authors would like to acknowledge funding by Nanosystems Initiative Munich (NIM) and thank the Center for NanoScience (CeNS) and the Augsburg Centre for Innovative Technologies (ACIT) for additional financial support for this project. We thank Rudolf Herrmann and Matthias Schneider for fruitful discussions and Alistair Ireland for proof reading. 


\section{References}

(1) Kafshgari, M.; Harding, F.; Voelcker, N. Insights into Cellular Uptake of Nanoparticles. Curr. Drug Deliv. 2015, 12 (1), 63-77. https://doi.org/10.2174/1567201811666140821110631.

(2) Pülat, H.; Karaköse, O.; Duran, A.; Zihni, I.; Özcelik, K. Ç.; Çalta, A. F.; Eken, H. Chondrosarcoma Located in the Costa: A Case Report. Surg. Chronicles 2016, 21 (3), 148-150. https://doi.org/10.1039/c0cs00003e.

(3) Treuel, L.; Jiang, X.; Nienhaus, G. U. New Views on Cellular Uptake and Trafficking of Manufactured Nanoparticles. J. R. Soc. Interface 2013, 10 (82), 20120939. https://doi.org/10.1098/rsif.2012.0939.

(4) Rejman, J.; Oberle, V.; Zuhorn, I. S.; Hoekstra, D. Size-Dependent Internalization of Particles via the Pathways of Clathrin- and CaveolaeMediated Endocytosis. Biochem. J. 2004, 377 (Pt 1), 159-169. https://doi.org/10.1042/BJ20031253.

(5) Römer, W.; Berland, L.; Chambon, V.; Gaus, K.; Windschiegl, B.; Tenza, D.; Aly, M. R. E.; Fraisier, V.; Florent, J.-C.; Perrais, D.; et al. Shiga Toxin Induces Tubular Membrane Invaginations for Its Uptake into Cells. Nature 2007, 450 (7170), 670-675. https://doi.org/10.1038/nature05996.

(6) Strobl, F. G.; Seitz, F.; Westerhausen, C.; Reller, A.; Torrano, A.; Bräuchle, C.; Wixforth, A.; Schneider, M. F. Intake of Silica Nanoparticles by Giant Lipid Vesicles : Influence of Particle Size and Thermodynamic Membrane State. Beilstein J. Nanotechnol. 2014, 5, 2468-2478.

https://doi.org/10.3762/bjnano.5.256.

(7) Yamashita, K.; Yoshioka, Y.; Higashisaka, K.; Mimura, K.; Morishita, Y.; Nozaki, M.; Yoshida, T.; Ogura, T.; Nabeshi, H.; Nagano, K.; et al. Silica and Titanium Dioxide Nanoparticles Cause Pregnancy Complications in Mice. Nat. Nanotechnol. 2011, 6 (5), 321-328. https://doi.org/10.1038/nnano.2011.41.

(8) Cho, M.; Cho, W. S.; Choi, M.; Kim, S. J.; Han, B. S.; Kim, S. H.; Kim, H. O.; Sheen, Y. Y.; Jeong, J. The Impact of Size on Tissue Distribution and Elimination by Single Intravenous Injection of Silica Nanoparticles. Toxicol. Lett. 2009, 189 (3), 177-183. https://doi.org/10.1016/j.toxlet.2009.04.017. 
(9) Mamaeva, V.; Sahlgren, C.; Lindén, M. Mesoporous Silica Nanoparticles in Medicine-Recent Advances. Advanced Drug Delivery Reviews. 2013, pp 689702. https://doi.org/10.1016/j.addr.2012.07.018.

(10) Barbu, E.; Molnàr, É.; Tsibouklis, J.; Górecki, D. C. The Potential for Nanoparticle-Based Drug Delivery to the Brain: Overcoming the Blood-brain Barrier. Expert Opin. Drug Deliv. 2009, 6 (6), 553-565. https://doi.org/10.1517/17425240902939143.

(11) Gao, K.; Jiang, X. Influence of Particle Size on Transport of Methotrexate across Blood Brain Barrier by Polysorbate 80-Coated Polybutylcyanoacrylate Nanoparticles. Int. J. Pharm. 2006, 310 (1-2), 213-219. https://doi.org/10.1016/j.jpharm.2005.11.040.

(12) Hanada, S.; Fujioka, K.; Inoue, Y.; Kanaya, F.; Manome, Y.; Yamamoto, K. Cell-Based in Vitro Blood-Brain Barrier Model Can Rapidly Evaluate Nanoparticles' Brain Permeability in Association with Particle Size and Surface Modification. Int. J. Mol. Sci. 2014, 15 (2), 1812-1825.

https://doi.org/10.3390/ijms15021812.

(13) Deserno, M.; Gelbart, W. Adhesion and Wrapping in Colloid- Vesicle Complexes. J. Phys. Chem. B 2002, 106, 5543-5552. https://doi.org/10.1021/jp0138476.

(14) Wittmann, C. G.; Kamenac, A.; Strobl, F. G.; Czubak, D.; Wixforth, A.; Westerhausen, $C$. lonic Strength and the Supporting Material Strongly Influence the Adhesion of Silica to Supported Lipid Bilayers. Adv. Biosyst. 2018, 2 (8), 1800087. https://doi.org/10.1002/adbi.201800087.

(15) Angelova, M. I.; Soléau, S.; Méléard, P.; Faucon, F.; Bothorel, P. Preparation of Giant Vesicles by External AC Electric Fields. Kinetics and Applications. In Trends in Colloid and Interface Science Vl; Helm, C., Lösche, M., Möhwald, H., Eds.; Progress in Colloid \& Polymer Science; Steinkopff, 1992; Vol. 89, pp 127-131. https://doi.org/10.1007/BFb0116295.

(16) Lipowsky, R.; Döbereiner, H.-G. Vesicles in Contact with Nanoparticles and Colloids. Europhys. Lett. 1998, 43 (2), 219-225. https://doi.org/10.1209/epl/i1998-00343-4.

(17) Verma, A.; Stellacci, F. Effect of Surface Properties on Nanoparticle-Cell 
Interactions. Small 2010, 6 (1), 12-21. https://doi.org/10.1002/smll.200901158.

(18) Ford, M. G. J.; Mills, I. G.; Peter, B. J.; Vallis, Y.; Praefcke, G. J. K.; Evans, P. R.; McMahon, H. T. Curvature of Clathrin-Coated Pits Driven by Epsin. Nature 2002, 419 (6905), 361-366. https://doi.org/10.1038/nature01020.

(19) Jaskiewicz, K.; Larsen, A.; Schaeffel, D.; Koynov, K.; Lieberwirth, I.; Fytas, G.; Landfester, K.; Kroeger, A. Incorporation of Nanoparticles into Polymersomes: Size and Concentration Effects. ACS Nano 2012, 6 (8), 7254-7262. https://doi.org/10.1021/nn302367m.

(20) Le Bihan, O.; Bonnafous, P.; Marak, L.; Bickel, T.; Trépout, S.; Mornet, S.; De Haas, F.; Talbot, H.; Taveau, J.-C.; Lambert, O. Cryo-Electron Tomography of Nanoparticle Transmigration into Liposome. J. Struct. Biol. 2009, 168 (3), 419425. https://doi.org/10.1016/j.jsb.2009.07.006.

(21) Dietrich, C.; Angelova, M.; Pouligny, B. Ahesion of Latex Spheres to Giant Phospholipid Vesicles: Statics and Dynamics. J. Phys. I/ Fr. 1997, 7, 16511682 .

(22) Deserno, M.; Gelbart, W. Adhesion and Wrapping in Colloid- Vesicle Complexes. J. Phys. Chem. B 2002, 106, 5543-5552. https://doi.org/10.1021/jp0138476.

(23) Evans, E.; Heinrich, V.; Ludwig, F.; Rawicz, W. Dynamic Tension Spectroscopy and Strength of Biomembranes. Biophys. J. 2003, 85 (4), 2342-2350. https://doi.org/10.1016/S0006-3495(03)74658-X.

(24) Akimov, S. A.; Volynsky, P. E.; Galimzyanov, T. R.; Kuzmin, P. I.; Pavlov, K. V; Batishchev, O. V. Pore Formation in Lipid Membrane I: Continuous Reversible Trajectory from Intact Bilayer through Hydrophobic Defect to Transversal Pore. Sci. Rep. 2017, 7 (1), 12152. https://doi.org/10.1038/s41598-017-12127-7.

(25) Akimov, S. A.; Volynsky, P. E.; Galimzyanov, T. R.; Kuzmin, P. I.; Pavlov, K. V.; Batishchev, O. V. Pore Formation in Lipid Membrane II: Energy Landscape under External Stress. Sci. Rep. 2017, 7 (1), 12509. https://doi.org/10.1038/s41598-017-12749-x.

(26) Sandre, O.; Moreaux, L.; Brochard-Wyart, F. Dynamics of Transient Pores in Stretched Vesicles. Proc. Natl. Acad. Sci. 1999, 96 (19), 10591-10596. 
https://doi.org/10.1073/pnas.96.19.10591.

(27) Karatekin, E.; Sandre, O.; Guitouni, H.; Borghi, N.; Puech, P.-H.; BrochardWyart, F. Cascades of Transient Pores in Giant Vesicles: Line Tension and Transport. Biophys. J. 2003, 84 (3), 1734-1749. https://doi.org/10.1016/S00063495(03)74981-9.

(28) Bahrami, A. H.; Raatz, M.; Agudo-Canalejo, J.; Michel, R.; Curtis, E. M.; Hall, C. K.; Gradzielski, M.; Lipowsky, R.; Weikl, T. R. Wrapping of Nanoparticles by Membranes. Adv. Colloid Interface Sci. 2014, 208, 214-224.

https://doi.org/10.1016/j.cis.2014.02.012.

(29) Marsh, D. Handbook of Lipid Bilayers, Second Edition; CRC Presse, 2013.

(30) Helfrich, W. Elastic Properties of Lipid Bilayers. Zeitschrift fur Naturforsch. C 1973, 28 (11), 693-703.

(31) Dagan, Z.; Weinbaum, S.; Pfeffer, R. An Infinite-Series Solution for the Creeping Motion through an Orifice of Finite Length. J. Fluid Mech. 1982, 115 (1), 505. https://doi.org/10.1017/S0022112082000883.

(32) Anas, I. What Are the True Values of the Bending Modulus of Simple Lipid Bilayers? Chem Phys. Lipids 2015, 20 (5), 113-130. https://doi.org/10.1016.

(33) Rawicz, W.; Olbrich, K. C.; McIntosh, T.; Needham, D.; Evans, E. Effect of Chain Length and Unsaturation on Elasticity of Lipid Bilayers. Biophys. J. 2000, 79 (1), 328-339. https://doi.org/10.1016/S0006-3495(00)76295-3.

(34) Nagle, J. F.; Pan, J.; Tristram-nagle, S.; Kuc, N. Temperature Dependence of Structure, Bending Rigidity, and Bilayer Interactions of Dioleoylphosphatidylcholine Bilayers. Biophys. J. 2008, 94 (1), 117-124. https://doi.org/10.1529/biophysj.107.115691.

(35) Anderson, T. H.; Min, Y.; Weirich, K. L.; Zeng, H.; Fygenson, D.; Israelachvili, J. N. Formation of Supported Bilayers on Silica Substrates. Langmuir 2009, 25 (12), 6997-7005. https://doi.org/10.1021/la900181c.

(36) Wittmann, C. G.; Kamenac, A.; Strobl, F. G.; Czubak, D.; Wixforth, A.; Westerhausen, $C$. Ionic Strength and the Supporting Material Strongly Influence the Adhesion of Silica to Supported Lipid Bilayers. Adv. Biosyst. 2018. 
(37) Le Bihan, O.; Bonnafous, P.; Marak, L.; Bickel, T.; Trépout, S.; Mornet, S.; De Haas, F.; Talbot, H.; Taveau, J.-C.; Lambert, O. Cryo-Electron Tomography of Nanoparticle Transmigration into Liposome. J. Struct. Biol. 2009, 168 (3), 419 425. https://doi.org/10.1016/j.jsb.2009.07.006.

(38) Michel, R.; Kesselman, E.; Plostica, T.; Danino, D.; Gradzielski, M. Internalization of Silica Nanoparticles into Fluid Liposomes : Formation of Interesting Hybrid Colloids. 2014, No. 126, 12649-12653. https://doi.org/10.1002/ange.201406927.

(39) Strobl, F. G.; Seitz, F.; Westerhausen, C.; Reller, A.; Torrano, A. a; Bräuchle, C.; Wixforth, A.; Schneider, M. F. Intake of Silica Nanoparticles by Giant Lipid Vesicles: Influence of Particle Size and Thermodynamic Membrane State. Beilstein J. Nanotechnol. 2014, 5, 2468-2478. https://doi.org/10.3762/bjnano.5.256.

(40) van der Wel, C.; Vahid, A.; Šarić, A.; Idema, T.; Heinrich, D.; Kraft, D. J. Lipid Membrane-Mediated Attraction between Curvature Inducing Objects. Sci. Rep. 2016, 6 (September), 32825. https://doi.org/10.1038/srep32825.

(41) Koltover, I.; Rädler, J. O.; Safinya, C. R. Membrane Mediated Attraction and Ordered Aggregation of Colloidal Particles Bound to Giant Phospholipid Vesicles. Phys. Rev. Lett. 1999, 82 (9), 1991-1994. https://doi.org/10.1103/PhysRevLett.82.1991.

(42) Michel, R.; Gradzielski, M. Experimental Aspects of Colloidal Interactions in Mixed Systems of Liposome and Inorganic Nanoparticle and Their Applications. Int. J. Mol. Sci. 2012, 13 (9), 11610-11642. https://doi.org/10.3390/ijms130911610.

(43) Betzer, O.; Chithrani, B. D.; Ghazani, A. A.; Chan, W. C. W.; Mukhopadhyay, S.; Barnés, C. M.; Haskel, A.; Short, S. M.; Katie, R.; Lippard, S. J. Determining the Size and Shape Dependence of Gold Nanoparticle Uptake into Mammalian Cells. Nano Lett. 2009, 19 (1), 662-668.

https://doi.org/10.1002/adma.200801393.Size-Dependent.

(44) Shang, L.; Nienhaus, K.; Nienhaus, G. U. Engineered Nanoparticles Interacting with Cells: Size Matters. J. Nanobiotechnology 2014, 12 (1), 1-11. https://doi.org/10.1186/1477-3155-12-5. 
(45) Zhang, S.; Gao, H.; Bao, G. Physical Principles of Nanoparticle Cellular Endocytosis. ACS Nano 2015, 9 (9), 8655-8671.

https://doi.org/10.1021/acsnano.5b03184.

(46) Rascol, E.; Devoisselle, J.-M.; Chopineau, J. The Relevance of Membrane Models to Understand Nanoparticles-cell Membrane Interactions. Nanoscale 2016, 8 (9), 4780-4798. https://doi.org/10.1039/C5NR07954C. 
Since our observations show that internalized, i.e. membrane coated particles cannot escape from the vesicle volume, we regard the uptake as a diffusive process through a membrane with an interior particle concentration $C_{\mathrm{i}}=0$. The total current of NP through the membrane with an area $A(t)$ can thus be described as

$$
I_{\text {int }}=\mathrm{d} N / \mathrm{d} t=C_{\mathrm{S}}(t) P A(t)
$$

We calculate the expression of diffusion current density $j(r)$ as a function of radial distance from the vesicle center $r$. For balanced flux, Fick's law of diffusion delivers:

$$
j(r)=\frac{I_{\mathrm{dif}}}{4 \pi r^{2}}=D \frac{\mathrm{d} C}{\mathrm{~d} r}
$$

where $D$ is the diffusion constant of the NP. Setting the boundary conditions $C(R)=$ $C_{\mathrm{S}}$ and $C(\infty)=C_{\infty}$, the diffusion current $I_{\mathrm{dif}}$ can be described as:

$$
I_{\text {dif }}=4 \pi D\left(C_{\infty}-C_{\mathrm{S}}\right) R
$$

Equilibrium implies the identity of diffusion- and internalizing currents $\left(I_{\text {int }}=I_{\text {dif }}=I\right)$ :

$$
\frac{\mathrm{d} A(t)}{\mathrm{d} t}=\frac{2 \sqrt{\pi} C_{\infty}^{\mathrm{A}} D P A(t)}{P \sqrt{A(t)}+2 \sqrt{\pi} D}
$$

The solution of eq. (4) is:

$$
A(t)=\frac{4 \pi D^{2} \mathfrak{B}\left(\frac{\sqrt{\frac{\frac{e^{D} \frac{}{D \sqrt{\pi}}-C_{\infty}^{A} P t}{D^{2}}}{D^{2}}}}{2 \sqrt{\pi}}\right)^{2}}{P^{2}}
$$

with $K$ as an integration constant and the Lambert-W-function $\mathfrak{M}$. 